\title{
Influence of sub-inhibitory antibiotics and flow condition on Staphylococcus aureus ATCC 6538 biofilm development and biofilm growth rate: BioTimer assay as a study model
}

\author{
Francesca Berlutti, Alessandra Frioni, Tiziana Natalizi, Fabrizio Pantanella and Piera Valenti
}

\begin{abstract}
Staphylococcus biofilm exhibits high antibiotic resistance and therapeutic doses of antibiotics are often sub-inhibitory. Whereas data are available on the effect of sub-inhibitory antibiotics on matrix formation, little is known on their influence on biofilm population. Here, using BioTimer Assay (BTA), a method developed to quantify biofilm population, the influence of subinhibitory gentamicin, ofloxacin and azithromycin on Staphylococcus aureus ATCC 6538 biofilm population in flow with respect to static condition was assessed. Antibiotics and flow condition increased biofilm population even if at different extent, depending on the antibiotic molecule. The greatest bacterial population was found in biofilm developed under flow condition in the presence of azithromycin. A significant increase in biofilm matrix was recorded for biofilm developed in the presence of antibiotics in flow with respect to static condition. The growth rates (GRs) of 24-h biofilm developed under the influence of antibiotics and flow condition were also evaluated using BTA and a specific mathematical model. Antibiotics and flow condition affected the GRs of 24-h biofilm even if at different extent. The lowest GR value was recorded for biofilm developed under flow condition in the presence of ofloxacin. Although further studies are needed, our data indicate that antibiotics and flow condition influenced biofilm development by increasing both bacterial population and matrix formation and affected the GRs of the developed biofilm. To the best of our knowledge, BTA is unique in allowing the calculation of the GRs of biofilm and it may be considered to be a useful study model to evaluate the activity of antibiofilm molecules.
\end{abstract}

The Journal of Antibiotics (2014) 67, 763-769; doi:10.1038/ja.2014.66; published online 28 May 2014

\section{INTRODUCTION}

Biofilm lifestyle is widespread in microbial world. Bacterial biofilms are responsible of a great part of clinical infections and particularly relevant are those related to bacteria of the genus Staphylococcus. ${ }^{1}$ Staphylococcus biofilm-mediated infections are difficult to treat as biofilm exhibits high antibiotic resistance. Therefore, therapeutic doses of antibiotics should be often considered as sub-inhibitory ${ }^{2,3}$ having an important role in microbial persistence and development of antibiotic resistance. Although data are available about the influence of antibiotics on the formation of Staphylococcus biofilm matrix, ${ }^{4-7}$ little is known on the influence of sub-inhibitory antibiotics on biofilm population because of the difficulties in its quantitative evaluation. ${ }^{8,9}$ As matter of the fact, to count bacteria in biofilm the bacteria are generally detached by vortexing or sonication and then the detached bacteria are counted using the CFU method. However, these sample manipulations do not ensure the detachment of all bacteria, thus often introducing a relevant bias in the obtained results. ${ }^{8,9}$

BioTimer Assay (BTA) is an indirect biological method useful to quantitatively evaluate bacterial population in biofilm without sample manipulation, thus overcoming the above mentioned difficulties. ${ }^{10,11}$ In addition, BTA was employed to assess the effect of sub-inhibitory antibiotics on Staphylococcus biofilm. ${ }^{5,10,11}$

Here, BTA was used to estimate the influence of sub-inhibitory antibiotics on the biofilm population of $S$. aureus. Since in vivo infections the bacterial biofilm is subjected to the flow of organic fluids, an experimental model allowing the medium flow was employed.

Recently, a renewal interest in assessing the growth rate (GR) of bacterial population has been shown, as it represents a pivotal parameter to determine the viability of bacteria under different conditions. ${ }^{12,13}$ Therefore, the GRs of S. aureus biofilm developed under the influence of sub-inhibitory antibiotics and flow condition were evaluated using BTA and a recently developed mathematical model. ${ }^{12}$

\section{MATERIALS AND METHODS \\ Strain}

S. aureus ATCC 6538 was maintained in Trypticase Soy broth (Becton Dickinson Italia S.p.A., Milan, Italy) with glycerol $25 \%$ at $-80^{\circ} \mathrm{C}$ and checked 
for purity on blood agar plates containing Columbia CNA agar (Difco Laboratories) and 5\% red sheep cells, before use. To prepare the inoculum, 10 isolated colonies on blood agar plates were taken and used to inoculate $10 \mathrm{ml}$ of $10 \%$ Brain Heart Infusion broth (BHI; Oxoid Ltd., Milan, Italy). The culture was incubated at $37^{\circ} \mathrm{C}$ for $4 \mathrm{~h}$ at 150 r.p.m. in an orbital shaker. After incubation, the $\mathrm{OD}_{600}$ of the broth culture was adjusted to $0.2 \pm 0.02$ corresponding to $\sim 5.0 \pm 0.5 \times 10^{7}$ CFUs per ml.

\section{Antibiotics}

Pure gentamicin, ofloxacin and azithromycin (Sigma-Aldrich, Milan, Italy) at final concentrations of 0.1 ( $1 / 64$ of the minimal biofilm inhibitory concentration (MBIC) value), 0.5 (1/16 of the MBIC value) and 0.25 (1/64 of the MBIC value) $\mu \mathrm{g} \mathrm{ml}{ }^{-1}$, respectively, were used. ${ }^{10}$ These sub-inhibitory doses reduced of about $70-75 \%$ the population of S. aureus ATCC 6538 biofilm as reported in Pantanella et al. ${ }^{10}$

\section{Experimental models}

The schematic diagram of the experimental models is shown in Figure 1. The experiments were carried out using two parallel models-that is, static and flow one; both were simultaneously performed under the same operation conditions. To grow S. aureus ATCC 6538 in biofilm, the strain was incubated in the presence of sterile glass beads (GBs; $5 \mathrm{~mm}$ of diameter). ${ }^{10,14}$
The static model consisted in a flask containing $40 \mathrm{ml}$ of $10 \%$ BHI broth without or with antibiotics containing sterile GBs that resulted completely immersed in the broth. The flasks were inoculated with $1.0 \pm 0.5 \times 10^{6} \mathrm{CFUs}$ per $\mathrm{ml}$ and incubated for $20 \mathrm{~h}$ at $37^{\circ} \mathrm{C}$ without shaking. After incubation, biofilms were recovered on colonized GBs. ${ }^{10,14}$

The flow model consisted in a flask containing the sterile 10\% BHI broth without or with antibiotics, a peristaltic pump (ISMATEC MPC, IDEX Health\&Science GmbH, Wertheim, Germany), a cylindrical glass flow reactor and a waste medium-collecting flask. The cylindrical flow reactor $(5 \mathrm{~cm}$ of diameter) contained a porous glass disk (6 $\mathrm{mm}$ in height) located at $4 \mathrm{~mm}$ from the bottom of the flow reactor on which the sterile GBs were placed. The flow reactors contained $40 \mathrm{ml}$ of $10 \%$ BHI broth without or with antibiotics and the GBs resulted completely immersed in the medium. The flow reactor was inoculated with $1.0 \pm 0.5 \times 10^{6} \mathrm{CFUs}$ per $\mathrm{ml}$ and incubated at $37^{\circ} \mathrm{C}$ for $4 \mathrm{~h}$ under static condition. Thereafter, the continuous flow was started and maintained at medium flow of $1 \mathrm{ml} \mathrm{min}^{-1}$ by the peristaltic pump for $16 \mathrm{~h}$. After incubation, biofilms were recovered on colonized GBs. ${ }^{10,14}$

\section{Quantification of viable biofilm population}

Biofilm population on GBs was determined with the BTA method that measures microbial metabolism. ${ }^{10,14}$ Briefly, BTA method employs BioTimerphenol red (BT-PR) medium whose color switches (red-to-yellow) due to S. aureus metabolism (Figure 1). ${ }^{10}$ The time required for BT-PR medium color

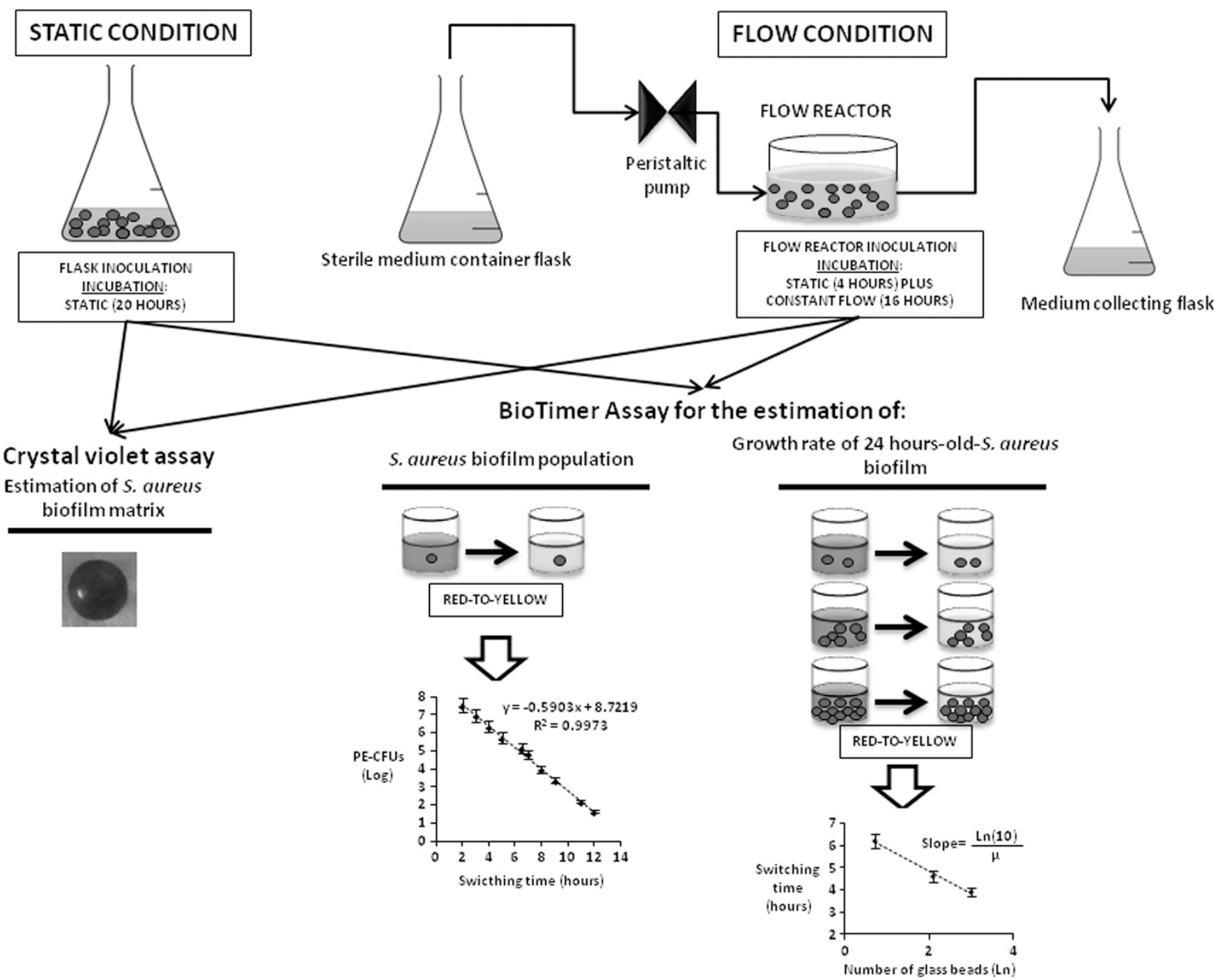

Figure 1 Schematic diagram of the experimental models. A full color version of this figure is available at The Journal of Antibiotics journal online. 
switch is correlated to initial bacterial concentration. Therefore, the time required for color switch determines the number of bacteria present in the sample at Time 0 through a specific correlation line. To draw the correlation line, serial twofold dilutions of overnight broth cultures in $1 \mathrm{ml}$ BT-PR reagent were performed in 24-well plates (Becton Dickinson Italia S.p.A.) and simultaneously counted using the CFU method. The equation and the linear correlation coefficient describing the correlation line were calculated on the whole data set and were: $y=-0.5903 \mathrm{x}+8.7219$ and $r^{2}=0.9973 .{ }^{10}$ To evaluate the viable biofilm population, colonized GBs were washed three times in sterile saline, immersed in $1 \mathrm{ml}$ of BT-PR medium and incubated at $37^{\circ} \mathrm{C}$. The time required for color switch of BT-PR medium was recorded and used to determine the number of viable bacteria in biofilm by the correlation line. As the correlation line correlated the time for color switch with the number of planktonic CFUs, the number of bacteria in biofilm was expressed as planktonic-equivalent CFUs (PE-CFUs). ${ }^{10,14}$

\section{Biofilm matrix detection}

Biofilm matrix was quantified using crystal violet (CV) assay. ${ }^{15}$ Briefly, colonized GBs were washed three times in sterile saline and soaked in aqueous solution of $\mathrm{CV}(1 \% \mathrm{w} / \mathrm{v})$. After $20 \mathrm{~min}$ of incubation at room temperature, stained GBs were washed to remove the excess of CV and airdried. Biofilm matrix was quantified by eluting $\mathrm{CV}$ with ethanol/acetone solution $(80: 20 \mathrm{v} / \mathrm{v})$ and by determining the absorbance of the eluted dye at $570 \mathrm{~nm}$ using a DU70 spectrophotometer (Beckman, Milan, Italy).

To observe biofilm matrix using epifluorescent microscopy, glass coverslips ( $5 \mathrm{~mm}$ of diameter) were placed in the flasks and flow reactors inoculated with S. aureus as above described. After incubation, colonized glass coverslips were washed twice with distilled water and stained using BacLight LIVE/DEAD viability probe (Molecular Probes, Life Technology, Monza, Italy) prepared according to the manufacturer's instructions. After $15 \mathrm{~min}$ of incubation in the dark, viable (stained green) and non-viable cells (stained red) were observed by using fluorescent optical microscopy (Leitz, Dialux 20 EB, Leica Microsystems Srl, Milan, Italy). Microscopic observations were performed at least five times in independent experiments.

\section{GR calculation}

To evaluate the GRs, after incubation, the colonized GBs were washed three times in sterile saline. Different amounts of colonized GBs (2, 8 and 20 GBs) were immersed in $3 \mathrm{ml}$ of BT-PR medium in the absence or in the presence of sub-inhibitory antibiotics and incubated at $37^{\circ} \mathrm{C}$. The time required for color switch of BT-PR medium was recorded and plotted versus the Ln of the number of colonized GBs. The relation between the time of BT-PR color switch and the Ln of the number of colonized GBs was described by linear equation:

$$
y=a x+b
$$

where the slope (a) of the line corresponded to the inverse of the specific GR $(\mu)$ of $S$. aureus biofilm according to the following equation: ${ }^{12}$

$$
a=\operatorname{Ln}(10) \mu^{-1}
$$

\section{Statistic}

Results were expressed as mean values \pm s.d.'s obtained from at least five independent experiments. Correlation lines were obtained by linear regression analysis and linear correlation coefficients were calculated from the equation: $r=(n \Sigma x y-\Sigma x \Sigma y) /(\operatorname{sqrt}((n \Sigma x 2-(\Sigma x) 2)(n \Sigma y 2-(\Sigma y) 2)))$. Statistical analysis was performed using Student's $t$-test. $P$-values $\leqslant 0.05$ and $\leqslant 0.01$ were considered significant at $95 \%$ and $99 \%$ of confidence, respectively.

\section{RESULTS}

Influence of sub-inhibitory antibiotics and flow condition on the development of $S$. aureus ATCC 6538 biofilm

To evaluate the influence of sub-inhibitory antibiotics and flow condition on S. aureus ATCC 6538 biofilm, biofilm population was estimated with the BTA protocol and biofilm matrix with CV assay and fluorescence microscopy (Tables 1 and 2; Figure 2).
Table 1 Influence of sub-inhibitory antibiotics and flow condition on

\begin{tabular}{|c|c|c|c|}
\hline \multirow[b]{2}{*}{ Treatment } & \multicolumn{2}{|c|}{$\begin{array}{c}\text { Log PE-CFUs as determined } \\
\text { by BTA }\end{array}$} & \multirow{2}{*}{$\begin{array}{l}\text { P-values (relative } \\
\text { to static condition) }\end{array}$} \\
\hline & Static condition & Flow condition & \\
\hline None (control) & $5.26 \pm 0.33$ & $6.03 \pm 0.21$ & 0.011 \\
\hline Gentamicin $(0.1 \mu \mathrm{g} \mathrm{ml}-1)$ & $5.08 \pm 0.37$ & $5.41 \pm 0.53^{a}$ & 0.0309 \\
\hline Ofloxacin $\left(0.5 \mu \mathrm{g} \mathrm{ml}^{-1}\right)$ & $5.14 \pm 0.45$ & $6.00 \pm 0.24$ & 0.0124 \\
\hline Azithromycin $\left(0.25 \mu \mathrm{g} \mathrm{ml}^{-1}\right)$ & $5.59 \pm 0.40$ & $6.53 \pm 0.20^{b}$ & 0.008 \\
\hline
\end{tabular}
Staphylococcus aureus ATCC 6538 biofilm population

Abbreviations: BTA, BioTimer Assay; PE-CFU, planktonic-equivalent CFU.

$\mathrm{PE}-\mathrm{CFUs}$ as determined by BTA were evaluated after $24 \mathrm{~h}$ of incubation. Values are expressed as the mean values and derived from at least five independent experiments. Statistical significance was calculated by Student's $t$-test. $P$-values $<0.05$ are significant at $95 \%$ of confidence; $P$-values $<0.01$ are significant at $99 \%$ of confidence.

astatistical significance relative to control $(P<0.05)$

bStatistical significance relative to control $(P<0.01)$

Table 2 Influence of sub-inhibitory antibiotics and flow condition on

\begin{tabular}{|c|c|c|c|}
\hline \multirow[b]{2}{*}{ Treatment } & \multicolumn{2}{|c|}{$\begin{array}{c}\text { Crystal violet } \\
\left(O D_{595}\right)\end{array}$} & \multirow{2}{*}{$\begin{array}{c}\text { P-values } \\
\text { (relative to static } \\
\text { condition) }\end{array}$} \\
\hline & Static condition & Flow condition & \\
\hline None (control) & $0.572 \pm 0.148$ & $0.4686 \pm 0.153$ & 0.448 \\
\hline Gentamicin $\left(0.1 \mu \mathrm{g} \mathrm{ml}^{-1}\right)$ & $0.667 \pm 0.181$ & $0.982 \pm 0.275^{a}$ & 0.0491 \\
\hline Ofloxacin $\left(0.5 \mu \mathrm{g} \mathrm{ml}^{-1}\right)$ & $0.339 \pm 0.032^{b}$ & $0.411 \pm 0.099$ & 0.0291 \\
\hline Azithromycin $\left(0.25 \mu \mathrm{g} \mathrm{ml}^{-1}\right)$ & $0.319 \pm 0.074$ & $0.777 \pm 0.141$ & 0.0004 \\
\hline
\end{tabular}
Staphylococcus aureus ATCC 6538 biofilm matrix development

Biofilm matrix was evaluated after $24 \mathrm{~h}$ of incubation. Crystal violet eluted from biofilm matrix is expressed as the mean values of $\mathrm{OD}_{595}$ deriving from at least five independent experiments. Statistic significance was calculated by Student's t-test. $P$-values $<0.05$ are significant at $95 \%$ of confidence, $P$-values $<0.01$ are significant at $99 \%$ of confidence.

a Statistical significance relative to control $(P<0.05)$.

aStatistical significance relative to control $(P<0.05)$.
bStatistical significance relative to control $(P<0.01)$.

Concerning biofilm population, the number of bacterial cells was significantly higher under flow than static condition both in the absence and in the presence of antibiotics (Table 1). Considering the data obtained under flow condition, biofilm population was differently affected by antibiotics as compared with control (Table 1). In particular, gentamicin significantly reduced and azithromycin significantly increased the biofilm population with respect to control.

The influence of antibiotics and flow condition on matrix formation was assessed through $\mathrm{CV}$ assay and microscopic observations (Table 2 and Figure 2). The CV assay showed that in the absence of sub-inhibitory antibiotics, similar values were recorded under static and flow conditions. On the contrary, the addition of sub-inhibitory antibiotics induced significant increase in biofilm matrix under flow with respect to static condition (Table 2). Representative images of epifluorescence observations are reported in Figure 2 and confirmed that biofilm matrix increased under the combined influence of subinhibitory antibiotics and flow condition. Indeed, single cells and small aggregates surrounded by a thin layer of matrix were observed under static condition both in the absence (Figures $2 \mathrm{a}$ and $\mathrm{b}$ ) and in the presence of antibiotics (Figures $2 \mathrm{c}$, e and g). On the contrary, under the combined influence of sub-inhibitory antibiotics and flow condition numerous and large aggregates surrounded by a thick layer of matrix were noticed (Figures $2 \mathrm{~d}, \mathrm{f}$ and $\mathrm{h}$ ). 

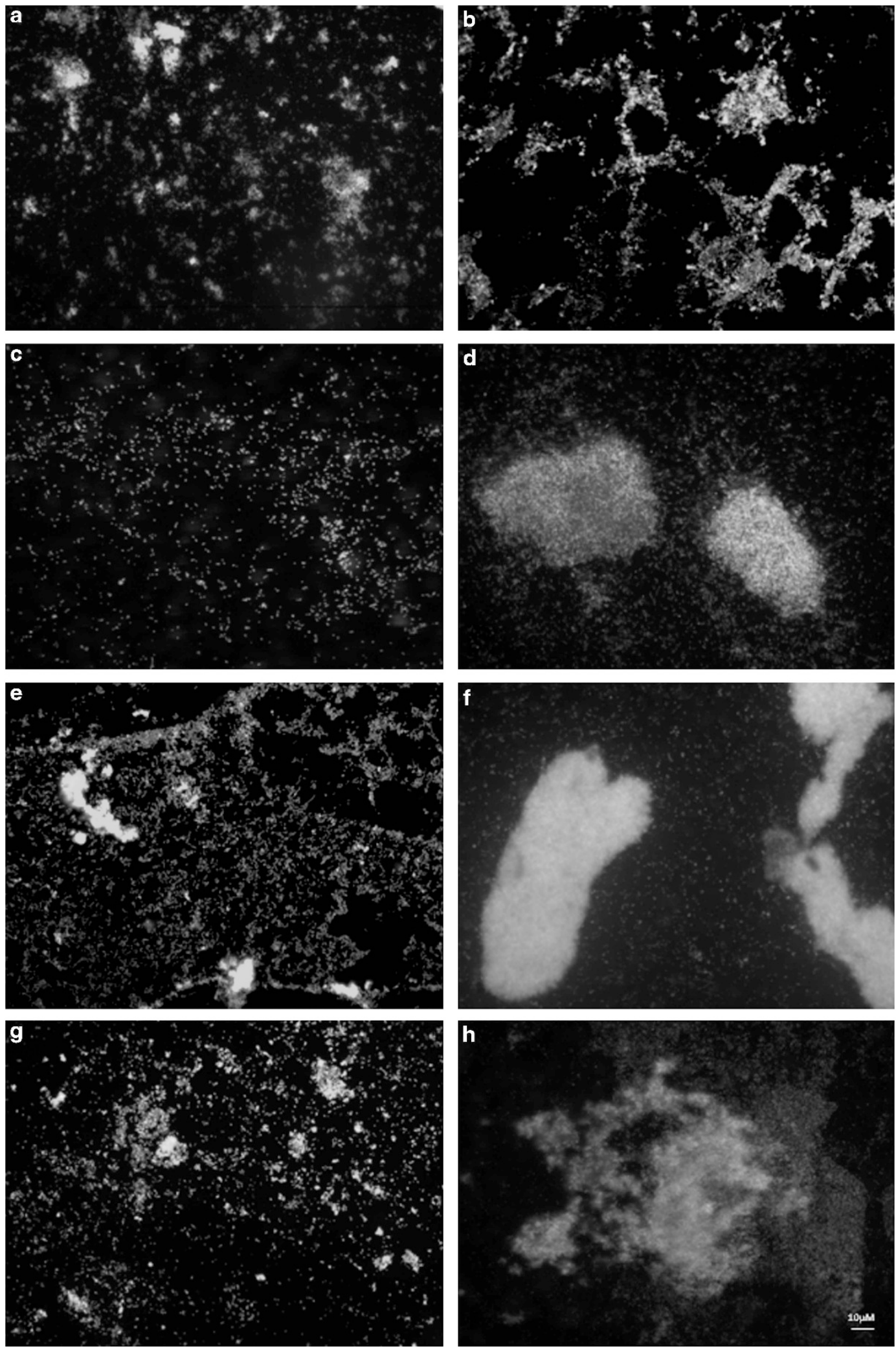

Figure 2 Micrographs of Staphylococcus aureus ATCC 6538 biofilm grown under different experimental conditions (epifluorescence staining). S. aureus biofilm was developed under static (a, $\mathbf{c}, \mathbf{e}, \mathbf{g})$ and flow conditions $(\mathbf{b}, \mathbf{d}, \mathbf{f}, \mathbf{h})$ in absence of sub-inhibitory antibiotic (a, b) or in the presence of subinhibitory gentamicin $(\mathbf{c}, \mathbf{d})$, ofloxacin $(\mathbf{e}, \mathbf{f})$ or azithromycin $(\mathbf{g}, \mathbf{h})$. Original magnifications $\times 600$. A full color version of this figure is available at The Journal of Antibiotics journal online. 

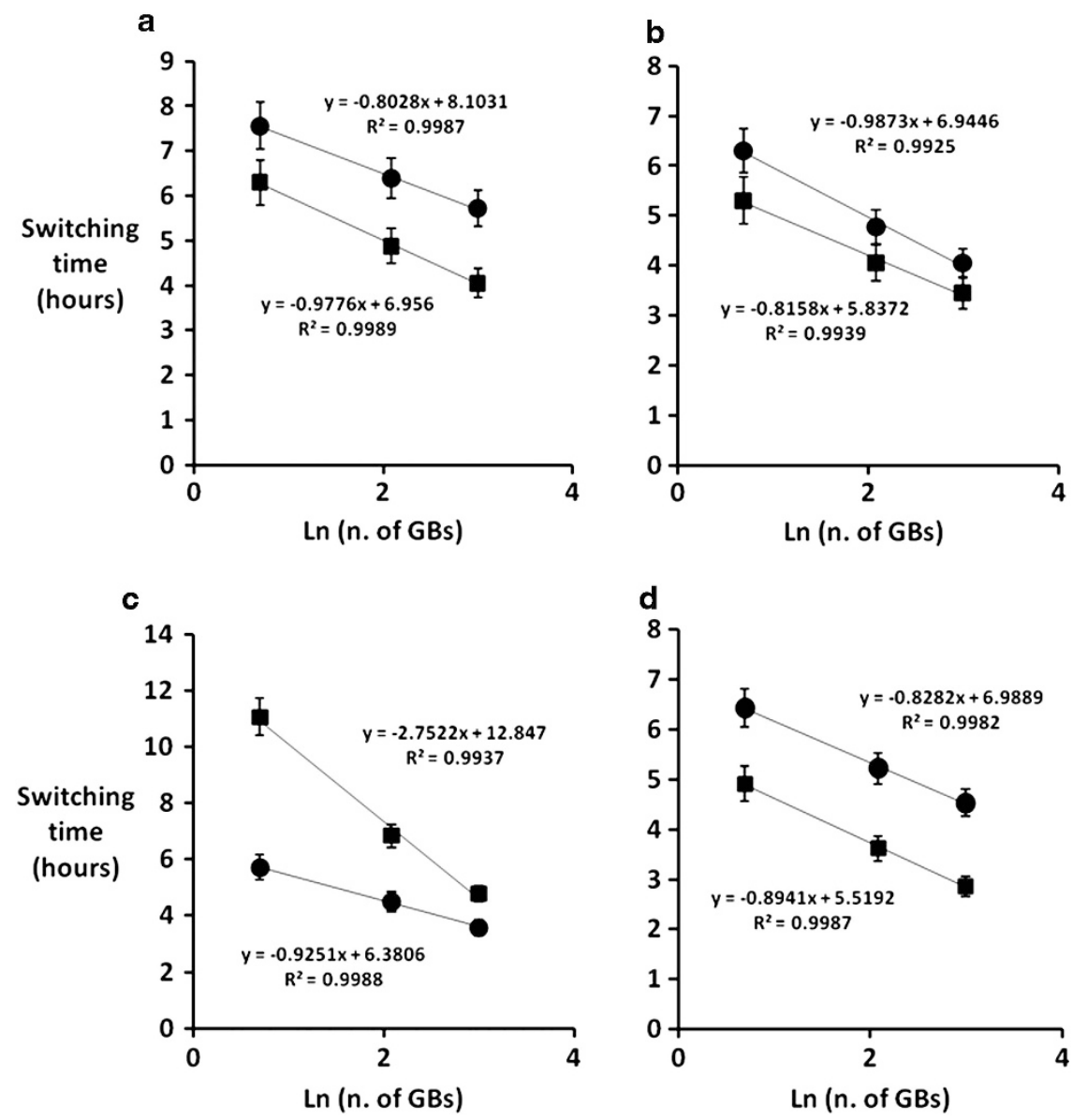

Figure 3 Relationships between the times required for color switch of BioTimer Assay reagents and the number of colonized GBs. BTA reagents were inoculated with different amount of GBs colonized with S. aureus ATCC 6538 biofilm developed in the absence of antibiotic (a) or in the presence of sub-inhibitory gentamicin (b), ofloxacin (c) and azithromycin (d) under flow (squares) or static conditions (circles).

GRs of $S$. aureus ATCC 6538 biofilm developed under the influence of sub-inhibitory antibiotics and flow condition

To evaluate the influence of antibiotics and flow condition on the GRs of 24-h-old biofilm, the BTA protocol and the mathematical model proposed by Welch et al. ${ }^{12}$ were employed. Briefly, increasing amounts of colonized GBs (that is, 2, 8 and $20 \mathrm{GBs}$ ) were used to inoculate the same volume of BT-PR reagent. When the switching times of inoculated BT-PR reagents were plotted versus the $\mathrm{Ln}$ of the number of colonized GBs, inverse relationships were found (Figure 3). The relationships were described by linear equations whose slope values were used to calculate the GR $(\mu)$ according to the equation: slope value $=\operatorname{Ln}(10) \mu^{-1}$ (Table 3$) .{ }^{12}$

In the absence of antibiotics, the GR value of 24-h-old biofilm was not affected by flow condition. The addition of antibiotics differently influenced the GRs in flow with respect to static condition depending on the antibiotic molecule. In particular, the GR value was significantly increased by gentamicin and significantly decreased by ofloxacin (Table 3). The lowest GR value was recorded for 24-h-old biofilm developed under the combined influence of ofloxacin and flow condition.

\section{DISCUSSION}

The antibiotic resistance of $S$. aureus biofilm is a great medical concern $^{1}$ and it is imperative to found molecule(s) active against biofilm. Unfortunately, standard susceptibility tests not
Table 3 Growth rates of Staphylococcus aureus ATCC 6538 biofilm developed under the influence of sub-inhibitory antibiotics and flow condition

\begin{tabular}{|c|c|c|c|}
\hline \multirow[b]{2}{*}{ Treatment } & \multicolumn{2}{|c|}{ Growth rates $\mu^{-1}$ (h) } & \multirow{2}{*}{$\begin{array}{c}\text { P-values } \\
\text { (relative to static } \\
\text { condition) }\end{array}$} \\
\hline & Static condition & Flow condition & \\
\hline None (control) & $2.6 \pm 1.1$ & $2.4 \pm 1.2$ & 0.782031 \\
\hline Gentamicin $\left(0.1 \mu \mathrm{g} \mathrm{ml}^{-1}\right)$ & $2.3 \pm 0.21$ & $2.8 \pm 0.1$ & 0.026561 \\
\hline Ofloxacin $\left(0.5 \mu \mathrm{g} \mathrm{ml} \mathrm{l}^{-1}\right)$ & $2.5 \pm 0.6$ & $0.8 \pm 0.2^{\mathrm{a}}$ & 0.009365 \\
\hline Azithromycin $\left(0.25 \mu \mathrm{g} \mathrm{m}^{-1}\right)$ & $2.8 \pm 0.6$ & $2.6 \pm 0.4$ & 0.638299 \\
\hline
\end{tabular}

The growth rates are expressed as the mean values deriving from at least five independent experiments. Statistical significance was calculated by Student's $t$-test. $P$-values $<0.05$ are significant at $95 \%$ of confidence; $P$-values $<0.01$ are significant at $99 \%$ of confidence. a Statistical significance relative to control $(P<0.01)$.

only are not predictive of the treatment outcome of biofilm-mediated infections ${ }^{16,17}$ but also are unable to evaluate the influence of sub-inhibitory antibiotics on biofilm development and GR.

Here, we employed BTA ${ }^{5,10,11,14,18}$ to evaluate the effect of subinhibitory antibiotics on the development of $S$. aureus biofilm population as well as on the GRs of developed biofilm (Figure 1). 
In our experimental model, three antibiotics were chosen as prototype of different mechanisms of action-that is, inhibition of protein synthesis by binding to $30 \mathrm{~S}$ (gentamicin) and to $50 \mathrm{~S}$ (azithromycin) ribosome subunits-and of DNA synthesis (ofloxacin). Moreover, to actually compare the effects of different antibiotics on biofilm development and GRs, we have employed sub-inhibitory doses that showed similar activity on S. aureus ATCC 6538 biofilm. ${ }^{10}$ As a matter of fact, identical sub-inhibitory doses of different antibiotics (for example, 1/16 of the MIC value) differently affect bacterial populations. $5,10,11,19$ Therefore, we chose antibiotic concentrations that reduced $S$. aureus biofilm population of $\sim 70-75 \% .{ }^{10}$ The experiments were carried out under two experimental conditions - that is, static and flow conditions. Our aim was to compare the static condition, typically used in literature, with the flow condition more similar to an in vivo situation in which the flow of organic fluids takes place. $^{20}$

Our data show that Staphylococcus population in biofilm was significantly greater under flow than static condition regardless of the antibiotics (Table 1). Moreover, under flow condition, the biofilm population was differently affected by sub-inhibitory antibiotics depending on the antibiotic molecule. On the contrary, the matrix formation was influenced only under the combined influence of antibiotics and flow condition (Table 2, Figure 2). Although a greater amount of dead cells were observed in flow with respect to static conditions (Figure 2), these data could be related to the limitations of the staining technique especially relevant in visualizing biofilm population. $^{21}$

These data confirm that biofilm development depends on the type of antibiotic given at sub-inhibitory doses. ${ }^{22-24}$ Concerning gentamicin, our results did not completely agree with those reported by Hess et al. ${ }^{5}$ who demonstrated that sub-inhibitory gentamicin increased S. aureus RN6390 but not S. aureus ATCC 25923 biofilm population on silk suture. However, these discrepancies may be related to the different $S$. aureus strains and to the material on which the biofilm was developed.?

The main novelty of the present paper relies on the possibility to estimate the GR of biofilm developed under the influence of subinhibitory antibiotics and flow condition using BTA. As a matter of fact, the classical microbiological techniques allow to determine the GR of a planktonic bacterial culture in exponential phase of growth as they are based on the determination of the net increase in bacterial population in an interval time. ${ }^{13}$ As the net increase in bacterial population in biofilm is $\approx 0,{ }^{10}$ the classical microbiological techniques are unable to evaluate the GRs of biofilm population. To the best of our knowledge, BTA is the sole method allowing evaluating the GR of bacteria in biofilm, thanks to the mathematical model described by Welch et al. ${ }^{12}$ We slightly modified the protocol and correlated the time for color switch of the same volumes of BTA reagent inoculated with the increasing amount of colonized GBs, and the number of the colonized GBs. Although the GBs were colonized at the same extent when cultured under the same experimental condition (Table 1), ${ }^{10}$ physiological heterogeneity in biofilm may occur thus varying the GR value at different regions of the biofilm within each GB. ${ }^{25,26}$ Therefore, to reduce the physiological heterogeneity we analyze biofilm samples constituted by 2, 8 or 20 GBs. In agreement with Welch et al., ${ }^{12}$ we obtained inverse relationships between the number of GBs and the switching time of BTA reagents (Figure 3, Table 3). When we extrapolated correlation lines, linear correlation coefficients were all $>0.99$ (Figure 3) indicating that the each sample of biofilm, constituted of 2,8 or 20 colonized GBs, as a whole, was homogeneous.
Interestingly, different GRs were recorded when the 24-h-old biofilm was developed under different conditions indicating that the culture conditions influenced not only biofilm population and matrix formation but also this pivotal parameter of biofilm viability.

Sub-inhibitory antibiotics differently influenced the GRs depending on antibiotic molecule (Table 3). Of note, ofloxacin was able to significantly reduce the GR of the 24-h-old biofilm with respect to control. This observation could be of interest since the recent interest on new molecules acting as inhibitors of bacterial DNA gyrase. ${ }^{27,28}$ Concerning ofloxacin, apparent contrasting results - that is, high biofilm population after $24 \mathrm{~h}$ of incubation (Table 1) and low GR of the 24-h-old biofilm (Table 3) -are shown. Although further experiments are needed, these data let us hypothesize that the GR may change during incubation, being higher at the beginning than at the end of incubation.

Taken together, these data suggest that the conditions occurring in vivo (that is, sub-inhibitory antibiotics and flow of organic fluids) could strongly influence the $S$. aureus biofilm development not only by increasing the matrix production ${ }^{4-7}$ but also by affecting the biofilm population and the GR values.

Although the presented results are limited to a single $S$. aureus strain and therefore cannot be generalized, our data suggest that to define the actual activity of antibiofilm molecules it should be necessary to consider not only their bactericidal/bacteriostatic property but also their influence on biofilm GR. ${ }^{17}$

In conclusion, BTA is able to highlight the influence of antibiotics and flow condition on biofilm population as well as on the GRs. This unique ability with respect to all other microbiological methods makes BTA a useful study model to evaluate the activity of antibiofilm molecules and as well as to study biofilm physiology.

\section{ACKNOWLEDGEMENTS}

We thank Luigi Rosa for his enthusiastic technical assistance.

1 Kiedrowski, M. R. \& Horswill, A. R. New approaches for treating staphylococcal biofilm infections. Ann. N.Y. Acad. Sci. 1241, 104-121 (2011).

2 del Pozo, J. L. \& Patel, R. The challenge of treating biofilm-associated bacterial infections. Clin. Pharmacol. Ther. 82, 204-209 (2007).

3 Molina-Manso, D. et al. In vitro susceptibility to antibiotics of staphylococci in biofilms isolated from orthopaedic infections. Int. J. Antimicrob. Agents 41, 521-523 (2013)

4 Rachid, S., Ohlsen, K., Witte, W., Hacker, J. \& Ziebuhr, W. Effect of subinhibitory antibiotic concentrations on polysaccharide intercellular adhesin expression in biofilm forming Staphylococcus epidermidis. Antimicrob. Agents Chemother. 44, 3357-3363 (2000).

5 Hess, D. J., Henry-Stanley, M. J. \& Wells, C. L. Gentamicin promotes Staphylococcus aureus biofilms on silk suture. J. Surg. Res. 170, 302-308 (2011).

6 Kaplan, J. B. Antibiotic-induced biofilm formation. Int. J. Artif. Organs. 34, 737-751 (2011).

7 Mirani, Z. A. \& Jamil, N. Effect of sub-lethal doses of vancomycin and oxacillin on biofilm formation by vancomycin intermediate resistant Staphylococcus aureus. J. Basic Microbiol. 51, 191-195 (2011).

8 Freitas, A. I., Vasconcelos, C., Vilanova, M. \& Cerca, N. Optimization of an automatic counting system for the quantification of Staphylococcus epidermidis cells in biofilms. J. Basic Microbiol. (e-pub ahead of print 20 May 2014; doi:10.1002/ jobm.201200603).

9 Pantanella, F., Valenti, P., Natalizi, T., Passeri, D. \& Berlutti, F. Analytical techniques to study microbial biofilm on abiotic surfaces: pros and cons of the main techniques currently in use. Ann. Ig. 25, 31-42 (2013).

10 Pantanella, F. et al. BioTimer Assay, a new method for counting Staphylococcus spp. in biofilm without sample manipulation applied to evaluate antibiotic susceptibility of biofilm. J. Microbiol. Methods 75, 478-484 (2008).

11 Wells, C. L., Henry-Stanley, M. J., Barnes, A. M., Dunny, G. M. \& Hess, D. J. Relation between antibiotic susceptibility and ultrastructure of Staphylococcus aureus biofilms on surgical suture. Surg. Infect. (Larchmt) 12, 297-305 (2011).

12 Welch, K., Cai, Y. \& Strømme, M. A method for quantitative determination of biofilm viability. J. Funct. Biomater. 3, 418-431 (2012).

13 Hall, B. G., Acar, H., Nandipati, A. \& Barlow, M. Growth rates made easy. Mol. Biol. Evol. 31, 232-238 (2014) 
14 Pantanella, F. et al. Quantitative evaluation of bacteria adherent and in biofilm on single-wall carbon nanotube-coated surfaces. Interdiscip. Perspect. Infect. Dis. 2011, 291513-291522 (2011)

15 Peeters, E., Nelis, H. J. \& Coenye, T. Comparison of multiple methods for quantification of microbial biofilms grown in microtiter plates. J. Microbiol. Methods 72, 157-165 (2008).

16 Giacometti, A. et al. Comparative efficacies of quinupristin-dalfopristin, linezolid, vancomycin, and ciprofloxacin in treatment, using the antibiotic-lock technique, of experimental catheter-related infection due to Staphylococcus aureus. Antimicrob. Agents Chemother. 49, 4042-4045 (2005).

17 Levin, B. R. \& Udekwu, K. I. Population dynamics of antibiotic treatment: a mathematical model and hypotheses for time-kill and continuous-culture experiments. Antimicrob. Agents Chemother. 54, 3414-3426 (2010).

18 Berlutti, F. et al. Quantitative evaluation of bacteria adherent to polyelectrolyte hema-based hydrogels. J. Biomed. Mater. Res. A 67A, 18-25 (2003).

19 Wang, Q. et al. Enhancement of biofilm formation by subinhibitory concentrations of macrolides in icaADBC-positive and -negative clinical isolates of Staphylococcus epidermidis. Antimicrob. Agents Chemother. 54, 2707-2711 (2010).

20 Navazesh, M. \& Kumar, S. K. Measuring salivary flow: challenges and opportunities. J. Am. Dent. Assoc. 139, 35S-40S (2008).
21 Netuschil, L., Auschill, T. M., Sculean, A. \& Arweiler, N. B. Confusion over live/dead stainings for the detection of vital microorganisms in oral biofilms-which stain is suitable? BMC Oral Health 11, 14-2 (2014).

22 Odenholt, I. Pharmacodynamic effects of subinhibitory antibiotic concentrations. Int. J. Antimicrob. Agents 17, 1-8 (2001).

23 Haddadin, R. N., Saleh, S., Al-Adham, I. S., Buultjens, T. E. \& Collier, P. J. The effect of subminimal inhibitory concentrations of antibiotics on virulence factors expressed by Staphylococcus aureus biofilms. J. Appl. Microbiol. 108, 1281-1291 (2010).

24 Frank, K. L., Reichert, E. J., Piper, K. E. \& Patel, R. In vitro effects of antimicrobial agents on planktonic and biofilm forms of Staphylococcus lugdunensis clinical isolates. Antimicrob. Agents Chemother. 51, 888-895 (2007).

25 Wentland, E. J., Stewart, P., Ching-Tsan, H. \& McFeters, G. A. Spatial variations in growth rate within Klebsiella pneumonia colonies and biofilm. Biotechnol. Prog. 12, 316-321 (1996).

26 Stewart, P. S. \& Franklin, M. J. Physiological heterogeneity in biofilms. Nat. Rev. Microbiol. 6, 199-210 (2008).

27 Odagiri, T. et al. Design, synthesis, and biological evaluations of novel 7-[7-amino-7methyl-5-azaspiro[2.4]heptan-5-yl]-8-methoxyquinolines with potent antibacterial activity against respiratory pathogens. J. Med. Chem. 56, 1974-1983 (2013).

28 Uria-Nickelsen, M. et al. Novel DNA gyrase inhibitors: microbiological characterisation of pyrrolamides. Int. J. Antimicrob. Agents 41, 28-35 (2013). 